\title{
Cross-talks between microRNAs and mRNAs in pancreatic tissues of streptozotocin-induced type 1 diabetic mice
}

\author{
CAIMING TIAN ${ }^{1,2^{*}}$, XIAOXI OUYANG ${ }^{3 *},{\text { QING } \mathrm{LV}^{1,4} \text {, YAOU ZHANG }}^{2}$ and WEIDONG XIE ${ }^{2}$ \\ ${ }^{1}$ Department of Chemistry, Tsinghua University, Beijing 100084; ${ }^{2}$ Shenzhen Key Laboratory of Health Science and \\ Technology, Division of Life Science and Health, Graduate School at Shenzhen, Tsinghua University, Shenzhen, \\ Guangdong 518055; ${ }^{3}$ Department of Health Inspection and Quarantine, School of Public Health, \\ Sun Yat-sen University, Guangzhou, Guangdong 510080; ${ }^{4}$ School of Life Sciences, \\ Tsinghua University, Beijing 100084, P.R. China
}

Received December 30, 2014; Accepted February 2, 2015

DOI: $10.3892 /$ br.2015.426

\begin{abstract}
Network cross-talks between microRNAs (miRNAs) and mRNAs may be useful to elucidate the pathological mechanisms of pancreatic islet cells in diabetic individuals. The aim of the present study was to investigate the cross-talks between miRNAs and mRNAs in pancreatic tissues of streptozotocin-induced diabetic mice through microarray and bioinformatic methods. Based on the miRNA microarray, 64 upregulated and 72 downregulated miRNAs were observed in pancreatic tissues in diabetic mice compared to the normal controls. Based on the mRNA microarrray, 507 upregulated mRNAs and 570 downregulated mRNAs were identified in pancreatic tissues in diabetic mice compared to the normal controls. Notably, there were 246 binding points between upregulated miRNA and downregulated mRNAs; simultaneously, there were 583 binding points between downregulated miRNA and upregulated mRNAs. These changed mRNA may potentially involve the following signaling pathways: Insulin secretion, pancreatic secretion, mammalian target of rapamycin signaling pathway, forkhead box $\mathrm{O}$ signaling pathway and phosphatidylinositol 3-kinase-protein kinase B signaling. The fluctuating effects of miRNAs and matched mRNAs indicated that miRNAs may have wide cross-talks with mRNAs in pancreatic tissues of type 1 diabetic mice. The cross-talks may play important roles in contributing to impaired islet functions and the development of diabetes. However, further functional validation should be conducted in the future.
\end{abstract}

Correspondence to: Dr Weidong Xie, Shenzhen Key Laboratory of Health Science and Technology, Division of Life Science and Health, Graduate School at Shenzhen, Tsinghua University, L402E, Tsinghua Campus, University Town of Xili, Shenzhen, Guangdong 518055, P.R. China

E-mail: xiewd@sz.tsinghua.edu.cn

* Contributed equally

Key words: diabetes, microRNA, microarray, network, islet cells

\section{Introduction}

Diabetes mellitus is a complex metabolic disease demonstrating impaired islet function $(1,2)$. Destruction of $\beta$-cells or the failure of these insulin-secreting cells to compensate for increased metabolic demand may account for the development of diabetes. Apoptosis, oxidative stress, mitochondrial dysfunction and endoplasmic reticulum (ER) stress responses, including c-Jun N-terminal kinase (JNK) activation, have been suggested as mechanisms for the changes of pancreatic $\beta$-cells in type 2 diabetes mellitus (T2DM) (3). T1DM is the result of autoimmune destruction of pancreatic $\beta$ cells (4). However, the mechanisms involved in islet dysfunction remain unclear.

microRNAs (miRNAs) play a key role in regulating islet function (5). Repression of mRNAs by miRNAs is an important mechanism for regulation of expression during cell fate specification, apoptosis and metabolism (6). In the previous study, miRNA-mRNA interactions were investigated in the pancreatic islets of spontaneously diabetic Goto-Kakizaki rats (7) and the human pluripotent stem cells based in vitro model of pancreatic differentiation (8). A number of key pairs of miRNA-mRNA were proposed to have significant roles in the pathogenesis of T2DM or islet development. However, few studies have reported the miRNA-mRNA cross-talk in T1DM.

In the present study, the cross-talks between miRNAs and mRNAs in the streptozotocin (STZ)-induced T1DM mice were investigated.

\section{Materials and methods}

Establishment of T1DMmouse model. Male National Institutes of Health (NIH) mice (age, 4 weeks) were purchased from the Guangdong Medical Laboratory Animal Center (Foshan, China). The animals were maintained in an environmentally controlled breeding room (temperature, $20 \pm 2^{\circ} \mathrm{C}$; humidity, $60 \pm 5 \%$, dark/light cycle, $12 \mathrm{~h}$ ). The mice were fed with standard laboratory chow and water ad libitum. The study was performed in strict accordance with the recommendations in the Guide for the Care and Use of Laboratory Animals of the Institutional Animal Care and Use Committee of Tsinghua University (Beijing, China). The protocol was approved by the 
Animal Welfare and Ethics Committee of Tsinghua University. Male NIH mice that were fasted for $24 \mathrm{~h}$ (weight, $18 \pm 2 \mathrm{~g}$ ) were used to induce the diabetic model by the intrainperitoneal injection of $100 \mathrm{mg} / \mathrm{kg} \mathrm{STZ}$ (Sigma-Aldrich, St. Louis, MO, USA). STZ was freshly prepared in $0.1 \mathrm{M}$ pre-cold citric acid buffer ( $\mathrm{pH} 4.5$ ). One week after the injection of STZ, diabetic animal models with fasting blood glucose of $>11.1 \mathrm{mmol} / \mathrm{l}$ were selected for further trials. Four weeks after STZ induction, the animals were sacrificed by cervical dislocation; pancreatic tissues were removed for further miRNA or RNA extractions. STZ-untreated mice served as the normal controls.

miRNA microarray analysis. The miRNA chip assay was conducted by the method of Exiqon miRNA array [The 7th generation of miRCURY ${ }^{\mathrm{TM}}$ LNA array (v.18.0), KangChen Bio-tech Inc., Shanghai, China]. Three different whole pancreatic tissues were freshly collected from three different diabetic mice. The three diabetic or normal mice had similar blood glucose and body weights. Three equal volumes $(\sim 0.33 \mathrm{ml})$ of different homogenates from whole pancreatic tissue of each mouse were mixed and subjected to miRNA and mRNA extracts. Total RNA was harvested using TRIzol (Invitrogen Life Technologies, Carlsbad, CA, USA) and the miRNeasy mini kit (Qiagen, Hilden, Germany) according to the manufacturer's instructions. Subsequent to having passed RNA quantity measurement using the NanoDrop 1000, the samples were labeled using the miRCURY ${ }^{\mathrm{TM}} \mathrm{Hy} 3^{\mathrm{TM}} / \mathrm{Hy} 5^{\mathrm{TM}}$ Power labeling kit and hybridized on the miRCURY ${ }^{\mathrm{TM}}$ LNA array (v.18.0). Following the washing steps, the slides were scanned using the Axon GenePix 4000B microarray scanner (Molecular Devices Corp., Sunnyvale, CA, USA). Scanned images were imported into the GenePix Pro 6.0 software (Axon Instruments, Molecular Devices Corp.) for grid alignment and data extraction. Replicated miRNAs were averaged and miRNAs with intensities $\geq 30$ in all the samples were chosen for calculating the normalization factor. Expressed data were normalized using the median normalization. Following normalization, differentially expressed miRNAs were identified through fold change filtering.

mRNA microarray analysis. Following the miRNA microarray analysis, collected total RNAs were also used to conduct the mRNA chip assay. The mRNA chip assay was conducted by the method of NimbleGen Mouse Gene Expression Microarrays (KangChen Bio-tech Inc.). Total RNA from each sample was quantified by the NanoDrop ND-1000 and RNA integrity was assessed by standard denaturing agarose gel electrophoresis. Total RNA of each sample was used for labeling and array hybridization as follows: i) Reverse transcription with the Invitrogen Superscript ds-cDNA synthesis kit; ii) ds-cDNA labeling with the NimbleGen one-color DNA labeling kit; iii) array hybridization using the NimbleGen Hybridization System followed by washing with the NimbleGen wash buffer kit; and iv) array scanning using the Axon GenePix 4000B microarray scanner. Scanned images (TIFF format) were imported into the NimbleScan software (version 2.5) for grid alignment and expression data analysis. Expression data were normalized through quantile normalization and the Robust Multichip Average (RMA) algorithm included in the NimbleScan software. The probe level (*_norm_RMA.pair) and gene level (*_RMA. calls) files were generated following normalization. All the gene level files were imported into the Agilent GeneSpring GX software (version 11.5.1) for further analysis. Genes that were $\geq 2$ out of 2 samples have values greater than or equal to the lower cut-off: 100.0 ('All Targets Value') were chosen for data analysis. Differentially expressed genes were identified through fold-change filtering. Pathway analysis and gene ontology (GO) analysis were applied to determine the roles of these differentially expressed genes played in these biological pathways or GO terms. Finally, hierarchical clustering was performed to show distinguishable gene expression profiling among samples.

miRNA-mRNA cross-talk analysis. Reverse expressions of miRNA and mRNA were selected (upregulation of miRNAs by $>2$ fold and downregulation of mRNAs by $<50 \%$; or downregulation of miRNAs by $<50 \%$ and upregulation of mRNAs by $>50 \%$ ) for miRNA-mRNA target prediction. Three different databases were used for miRNA target predictions: miRanda (www.microrna.org/microrna/getDownloads.do), miRBase (http://www.mirbase.org/) and TargetScan (www. targetscan.org/mmu_60/), and only those targets predicted by at least two databases were selected. Furthermore, based on the bioinformatics prediction, only miRNA target gene pairs with such opposing expression patterns were selected. Figures of miRNA-mRNA interactions were drawn by the cytoscape software (9). Selected mRNA was subjected to further hierarchical clustering analysis, pathway analysis (http://www. genome.jp/kegg/pathway.html) and GO analysis.

\section{Results}

miRNA microarray analysis. According to the miRNA chip report, 64 miRNAs showed $>2$-fold increase in pancreatic tissues of diabetic mice compared to the normal controls (Table I). However, 72 miRNAs showed $\geq 50 \%$ decrease in pancreatic tissues of diabetic mice compared to the normal controls (Table II). However, functions of the majority of miRNAs remained unclear.

mRNA microarray analysis. According to the gene chip report, 507 mRNAs were upregulated by $>2$-fold while 570 mRNAs were downregulated by $\geq 50 \%$ (available upon request). Among the upregulated mRNAs, certain genes from viral myocarditis, tight junction, pentose and glucuronate interconversions, autoimmune thyroid disease and cocaine addiction pathways appeared to be enhanced in the pancreatic tissues of diabetic mice (Table III). Among the downregulated mRNAs, certain genes from pancreatic secretion, endocrine and other factor-regulated calcium reabsorption, Alzheimer's disease, endometrial cancer, mammalian target of rapamycin (mTOR) signaling pathway, T1DM, neurotrophin signaling pathway, aminoacyl-tRNA biosynthesis, maturity onset diabetes of the young, oxidative phosphorylation and T2DM pathways were attenuated (Table IV). Gene expression analysis identified that pancreatic islet cells showed severe impairments of functions in STZ-induced diabetic mice. These impaired functions included abnormal expressions of insulin synthesis, glucose or energy metabolism, cell skeleton and apoptosis 
Table I. Upregulated miRNAs in pancreatic tissues of streptozotocin-induced diabetic mice compared to the normal controls.

Fold

miRNA names

(diabetes vs. normal)

miRNA names

Fold

\begin{tabular}{|c|c|c|c|}
\hline mmu-miR-5119 & 38.19127 & $m m u-m i R-132-5 p$ & 2.287934 \\
\hline$m m u-m i R-5130$ & 12.56928 & $m m u-m i R-137-5 p$ & 2.27541 \\
\hline$m c m v-m i R-m 88-1-5 p$ & 6.381325 & $m m u-m i R-3102-3 p$ & 2.267138 \\
\hline$m m u-m i R-881-3 p$ & 5.511145 & $m m u-m i R-1249-3 p$ & 2.256024 \\
\hline mmu-miR-697 & 4.16046 & mmu-miR-3070a-5p/mmu-miR-3070b-5p & 2.250164 \\
\hline mmu-miR-5622-3p & 4.079709 & mmu-miR-1953 & 2.229568 \\
\hline mти-тiR-323-3p & 3.751446 & mти-miR-3090-5p & 2.209983 \\
\hline$m m u-m i R-154-3 p$ & 3.667428 & $m m u-m i R-5115$ & 2.198546 \\
\hline$m m u-m i R-3058-3 p$ & 3.603779 & $m m u-m i R-653-3 p$ & 2.18967 \\
\hline$m m u-m i R-669 m-3 p$ & 3.579467 & $m m u-m i R-3095-3 p$ & 2.188949 \\
\hline$m m u-m i R-670-5 p$ & 3.384036 & $m m u-m i R-292-5 p$ & 2.169091 \\
\hline$m m u-m i R-491-5 p$ & 3.344572 & $m m u-m i R-3078-3 p$ & 2.164942 \\
\hline$m m u-m i R-296-3 p$ & 3.222892 & $m т и-m i R-5132-5 p$ & 2.164756 \\
\hline$m m u-m i R-139-5 p$ & 3.142319 & $m m u-m i R-5114$ & 2.157146 \\
\hline mmu-miR-5617-5p & 3.110791 & $m m u-m i R-700-5 p$ & 2.156858 \\
\hline mmu-miR-3102-5p & 3.086538 & mmu-miR-1956 & 2.148594 \\
\hline$m m u-m i R-668-3 p$ & 3.049351 & $m m u-m i R-361-3 p$ & 2.141795 \\
\hline$m m u-m i R-9-3 p$ & 3.029518 & $m m u-m i R-1247-5 p$ & 2.127108 \\
\hline$m m u-m i R-325-5 p$ & 2.750409 & $m m u-m i R-30 b-3 p$ & 2.118166 \\
\hline$m m u-m i R-490-5 p$ & 2.707229 & $m m u-m i R-3105-3 p$ & 2.115023 \\
\hline$m m u-m i R-337-3 p$ & 2.635188 & $m m u-m i R-363-5 p$ & 2.112184 \\
\hline$m m u-m i R-219-2-3 p$ & 2.591205 & $m m u-m i R-302 b-5 p$ & 2.10827 \\
\hline mmu-miR-3081-3p & 2.575025 & $m m u-m i R-5616-3 p$ & 2.105276 \\
\hline$m m u-m i R-201-3 p$ & 2.570188 & $m m u-m i R-369-3 p$ & 2.091376 \\
\hline mmu-miR-3109-5p & 2.563323 & $m m u-m i R-450 a-2-3 p$ & 2.085864 \\
\hline mmu-miR-705 & 2.540397 & $m m u-m i R-133 b-3 p$ & 2.076568 \\
\hline$m m u-m i R-194-2-3 p$ & 2.512103 & $m m u-m i R-133 a-3 p$ & 2.075921 \\
\hline$m m u-m i R-346-5 p$ & 2.429564 & $m g h v-m i R-M 1-8-3 p$ & 2.059848 \\
\hline$m g h v-m i R-M 1-5-3 p$ & 2.417169 & $m m u-m i R-712-3 p$ & 2.023976 \\
\hline$m m u-m i R-5120$ & 2.357418 & $m m u-m i R-7 b-3 p$ & 2.02032 \\
\hline$m m u-m i R-744-5 p$ & 2.320811 & $m m u-m i R-5110$ & 2.010067 \\
\hline mmu-miR-1231-3p & 2.307297 & mmu-miR-302a-3p & 2.003911 \\
\hline
\end{tabular}

miRNA and miR, microRNA.

genes. However, the factors that caused these wide changes of mRNAs remain to be discovered.

Cross-talks between miRNAs and mRNAs. Whether there were certain correlations between these miRNA and mRNA remained unclear, as miRNA have the potential to inhibit the expression of mRNAs. In the present study, the target genes of miRNAs were predicted. Notably, certain upregulated miRNAs were accompanied by certain downregulated targeted mRNAs, while some downregulated miRNAs were accompanied by some upregulated targeted mRNAs (Fig. 1). Through software prediction, it is easy to observe that 1-18 miRNAs can co-target one common gene and one miRNA can target 1-23 different genes. Therefore, miRNA may form a complicated network to regulate the gene expression. There are 246 binding points between upregulated
miRNA and downregulated mRNAs. Simultaneously, there are 583 binding points between downregulated miRNA and upregulated mRNAs.

Furthermore, the signaling pathway analysis regarding those changed targeted mRNAs and reversely changed miRNAs was conducted. The upregulated miRNAs were associated with the changes of the following signaling pathways: Transcriptional misregulation in cancer, dopaminergic synapse, insulin secretion, morphine addiction, alcoholism, endometrial cancer, Hippo signaling pathway, acute myeloid, leukemia, pancreatic secretion, dorso-ventral axis formation, mTOR signaling pathway, viral carcinogenesis, salivary secretion, forkhead box $\mathrm{O}$ (FoxO) signaling pathway, hepatitis $\mathrm{C}$, phosphatidylinositol 3-kinase (PI3K)-protein kinase B (Akt) signaling pathway and Gap junction pathways (Table V). Among these signaling pathways, insulin secretion, pancreatic 
Table II. Downregulated miRNAs in pancreatic tissues of streptozotocin-induced diabetic mice compared to the normal controls.

Fold

miRNA names (diabetes vs. normal)
Fold

miRNA names

mmu-miR-669k-5p

0.304525

0.010509

0.026857

mmu-miR-467h

0.088327

mmu-miR-410-5p

0.094329

0.112933

$m m u-m i R-466 q$

0.127823

mmu-miR-669f-3p

0.129326

mmu-miR-466i-3p

0.132707

mmu-miR-669a-3p/mmu-miR-669o-3p

0.137753

mmu-miR-466e-5p

0.144532

mmu-miR-143-3p

mmu-miR-28a-5p/mmu-miR-28c

0.1686

0.181654

0.188657

mmu-miR-669e-5p

0.19437

mти-miR-467g

0.198745

mmu-miR-1187

0.199611

mmu-miR-466c-5p

0.200232

mmu-miR-101a-3p/mmu-miR-101c

0.207186

mmu-miR-184-3p

0.216273

mmu-miR-7b-5p

0.218596

mmu-miR-467c-3p

0.228035

mmu-miR-669d-2-3p

0.228356

mmu-miR-126-5p

0.241717

mmu-miR-16-5p

0.251913

0.257212

0.259083

0.268486

0.269823

0.274186

0.2806

0.283552

0.284459

0.287447

0.289108

0.297498

0.298627
mmu-miR-466f-3p

mmu-miR-10b-5p

mmu-miR-669c-3p

mmu-let-7g-5p

mmu-miR-142-3p

mmu-miR-669f-5p

mmu-miR-19a-3p

mmu-miR-27a-3p

mmu-miR-5624-3p

mmu-miR-26a-5p

$m m u-m i R-30 b-5 p$

mmu-miR-466f

mmu-miR-32-3p

mmu-miR-802-5p

mmu-miR-669e-3p

mmu-miR-669p-3p

mmu-miR-758-5p

mmu-miR-669l-5p

mmu-miR-216a-5p

mmu-miR-15a-5p

mmu-miR-1948-5p

mmu-miR-200a-3p

mmu-miR-669a-3-3p

mmu-miR-669b-3p

mmu-let-7d-5p

mmu-miR-574-5p

mmu-miR-10a-5p

mmu-miR-466i-5p

mmu-miR-3082-5p

mmu-miR-148b-3p

mmu-miR-98-5p

mmu-miR-493-5p

mmu-let-7c-5p

mmu-miR-324-3p

mmu-miR-182-5p
0.313121

0.317253

0.320678

0.322723

0.335648

0.337821

0.338066

0.341027

0.355022

0.361653

0.371462

0.374962

0.37608

0.37718

0.382497

0.383678

0.384668

0.391643

0.396066

0.399025

0.400083

0.401934

0.421249

0.422497

0.429719

0.44261

0.445411

0.446784

0.450945

0.456944

0.461151

0.462133

0.479815

0.487674

0.494421

miRNA and miR, microRNA.

secretion, mTOR signaling pathway, FoxO signaling pathway and PI3K-Akt signaling pathway appeared to be closely associated with the development of diabetes. Other signaling pathways (transcriptional misregulation in cancer, dopaminergic synapse, morphine addiction, alcoholism, endometrial cancer, Hippo signaling pathway, acute myeloid leukemia, dorso-ventral axis formation, hepatitis $\mathrm{C}$ and Gap junction) require further validation on whether these pathways were directly or indirectly associated with the development of diabetes.

The downregulated miRNAs were associated with the changes of the following signaling pathways: Axon guidance,
Ras-proximate-1 (Rap1) signaling pathway, pentose and glucuronate interconversions, estrogen signaling pathway, melanogenesis, retrograde endocannabinoid signaling, nucleotide excision repair, Huntington's disease, and amino sugar and nucleotide sugar metabolism (Table VI). Among these signaling pathways, pentose and glucuronate interconversions, amino sugar and nucleotide sugar metabolism appeared to be enhanced in the pancreatic tissues of diabetic mice, as $K L$, $U G D H$ and phosphomannomutase 1 expression was upregulated. The Axon guidance, Rap1 signaling pathway, estrogen signaling pathways and nucleotide excision repair pathway 
Table III. Upregulated mRNA mainly involved in the following signaling pathways.

\begin{tabular}{lcl}
\hline Pathway & Enrichment score & \multicolumn{1}{c}{ Genes } \\
\hline Viral myocarditis & 2.323506 & CD28//H2-BL//H2-T23//MYH10//MYH13//MYH7B \\
Tight junction & 1.994797 & $F 11$ R//GNAI2//MYH10//MYH13//MYH7B//TJAP1//TJP2 \\
Pentose and glucuronate interconversions & 1.735135 & $D C X R / / K L / / U G D H$ \\
Autoimmune thyroid disease & 1.497729 & $C D 28 / / H 2-B L / / H 2-T 23 / / I F N A 7$ \\
Cocaine addiction & 1.350065 & $C R E B 5 / / G N A I 2 / / P D Y N$ \\
\hline
\end{tabular}

Table IV. Downregulated mRNAs mainly involve the following signaling pathways.

\begin{tabular}{|c|c|c|}
\hline Pathway & Enrichment score & Genes \\
\hline Pancreatic secretion & 3.298924 & $\begin{array}{l}\text { AMY2A4//ATP1A3//CCKAR//CPA1//CTRB1//GNAS// } \\
\text { ITPR2//KCNMA1//PNLIP//RAC1 }\end{array}$ \\
\hline $\begin{array}{l}\text { Endocrine and other factor-regulated } \\
\text { calcium reabsorption }\end{array}$ & 3.101448 & $\begin{array}{l}\text { ATP1A3//GNAS//KLK1B11//KLK1B24// } \\
\text { KLK1B26//KLK1B5//KLK1B9 }\end{array}$ \\
\hline Alzheimer's disease & 1.89058 & $\begin{array}{l}\text { APBB1//ATP5G1//CACNA1C//CASP9//ITPR2// } \\
\text { LPL//NDUFA11//NDUFA2//NDUFA6/NDUFC1//NDUFV3 }\end{array}$ \\
\hline Endometrial cancer & 1.864948 & CASP9//FOXO3//ILK//SOS1//TCF7L2 \\
\hline mTOR signaling pathway & 1.831442 & INS1//INS2//STK11//STRADA//VEGFB \\
\hline Type I diabetes mellitus & 1.646775 & CPE//H2-K1//H2-Q6//INS1//INS2 \\
\hline Neurotrophin signaling pathway & 1.575833 & $\begin{array}{l}\text { FOXO3//MAP2K5//NGFRAP1//NTRK2//PTPN11//RAC1// } \\
\text { SOS1//YWHAB }\end{array}$ \\
\hline Aminoacyl-tRNA biosynthesis & 1.486088 & CARS2//IARS2//SARS//TARS//YARS \\
\hline Maturity onset diabetes of the young & 1.475269 & INS1//INS2//NEUROD1 \\
\hline Oxidative phosphorylation & 1.350224 & $\begin{array}{l}\text { ATP5G1//ATP6V0A2//ATP6V1B2//NDUFA11//NDUFA2// } \\
\text { NDUFA6//NDUFC1//NDUFV3 }\end{array}$ \\
\hline Type II diabetes mellitus & 1.319886 & CACNAIC//HK2//INSI//INS2 \\
\hline
\end{tabular}

mTOR, mammalian target of rapamycin.

were associated with the regulation of actin cytoskeleton, cell adhesion, cell cycle and cell apoptosis.

Insulin or pancreatic secretion pathway. For the insulin or pancreatic secretion pathway, the miRNAs and mRNA pairs: $m m u-m i R-705 / A T P 1 A 3, m m u-m i R-450 a-2-3 p / C C K A R$, mmu-miR-30b-3p/GNAS, mmu-miR-139-5p/KCNMAl and $m m u-m i R-669 m-3 p / K C N M A 1$ may be involved in the potential cross-talks between miRNAs and mRNAs (Table VII).

FoxO signaling pathway. For the FoxO signaling pathway, the miRNAs and mRNA pairs: $m m u-m i R-323-3 p / m m u-m i R-$ 369-3p/SOS1, mmu-miR-302a-3p/STK11, mmu-miR-302b-3p/ mmu-miR-491-5p/GADD45A and mmu-miR-133a-3p/mmu$m i R-133 b-3 p / m m u-m i R-1956 / G A B A R A P L 1$ may be involved in the potential cross-talks between miRNAs and mRNAs (Table VIII).

PI3K-Akt signaling pathway. For the PI3K-Akt signaling pathway, the miRNAs and mRNA pairs: $m m u-m i R-139-5 p /$ GNB1, mmu-miR-881-3p/SPP1, mmu-miR-337-3p/YWHAB, mmu-miR-323-3p/mmu-miR-369-3p/SOS1, mmu-miR-1956/
PPP2RIB and mmu-miR-1956/mmu-miR-668-3p/mmu$m i R-668-3 p / P P P 2 R 2 A$ may be involved in the potential cross-talks (Table IX).

mTOR signaling pathway. For the mTOR signaling pathway, the miRNAs and mRNA pairs: $m m u-m i R-302 a-3 p / S T K 11$, mmu-miR-1953/STRADA and mmu-miR-302a-3p/RRAGD may be involved in the potential cross-talks (Table X).

Summary. As shown in the above results, there were certain promising cross-talks that mediated the development of diabetes but require further validation (Table XI).

\section{Discussion}

In human islet $\beta$-cells, the 10 most abundant miRNAs were miR-375, miR-7-5p, let-7f-5p, let-7a-5p, let-7b-5p, miR-27b-3p, $m i R-192-5 p, m i R-148 a-3 p, m i R-26 a-5 p$ and $m i R-127-3 p$ (10). STZ-induced diabetic mice showed reduced islet $\beta$-cells compared to normal controls as previously reported (11). In the present study, STZ-induced diabetic mice showed reduced let-7f-5p, let-7b-5p, let-7a-5p, miR-7b-5p, miR-7a-5p, 
A

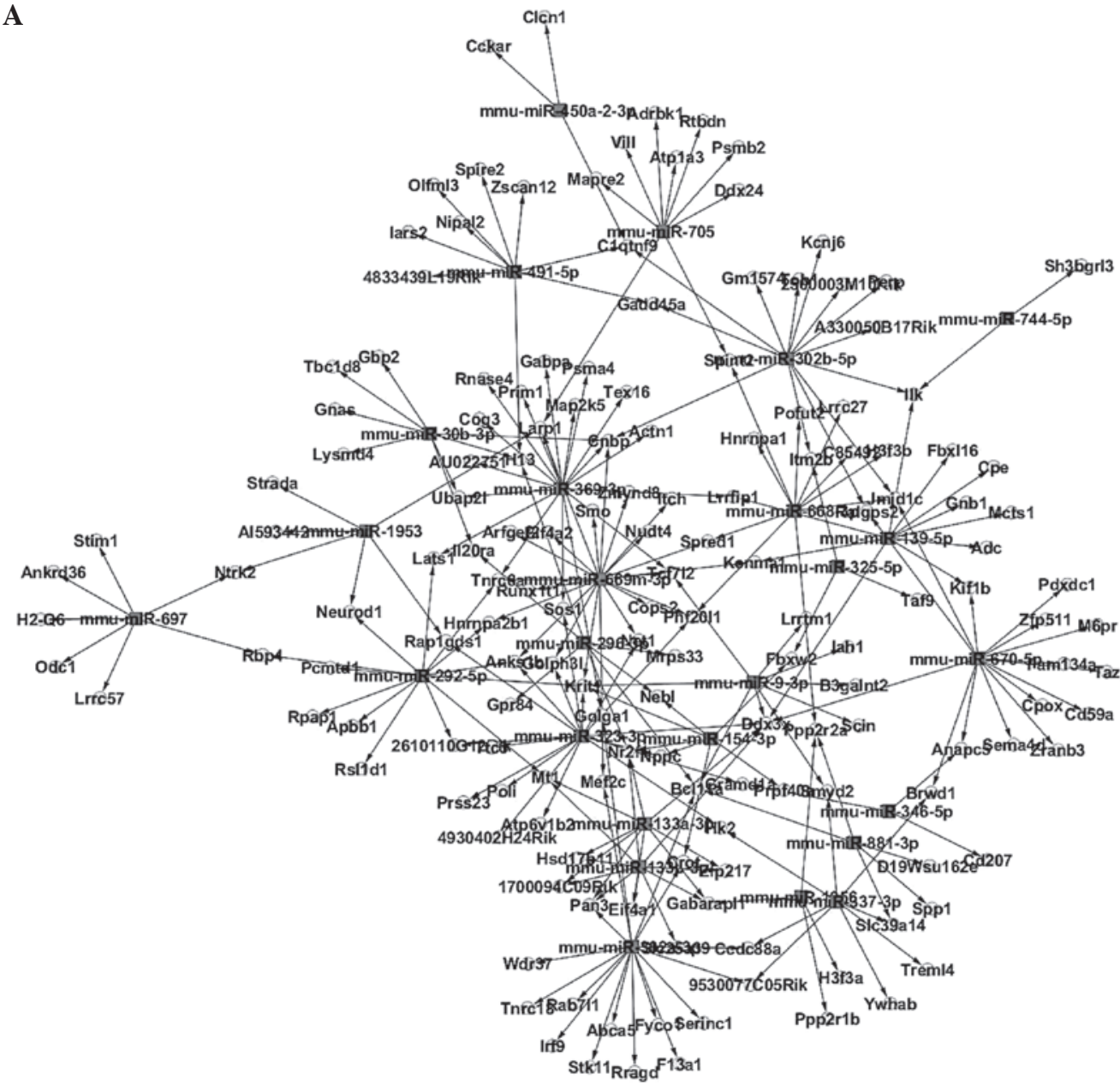

B

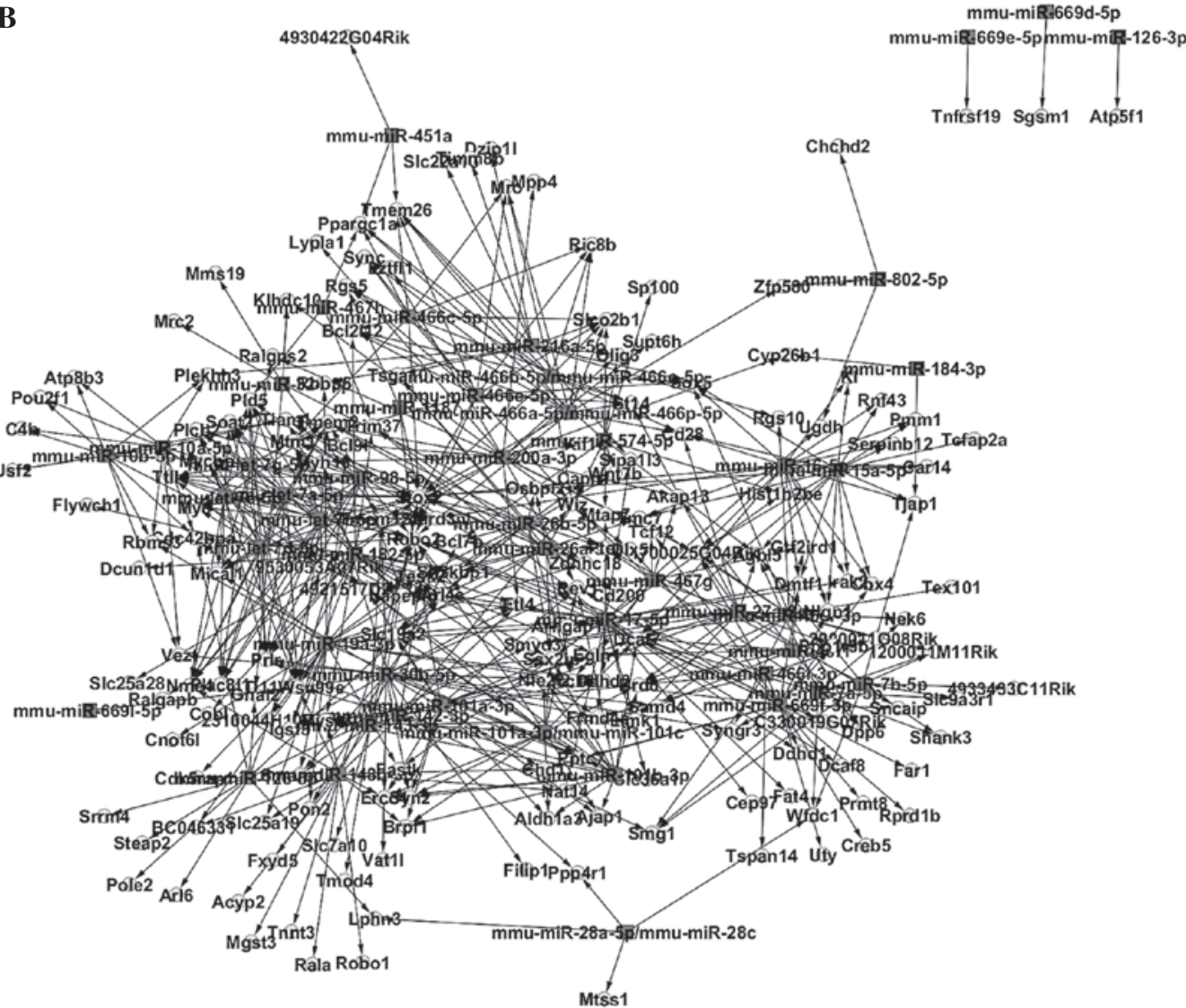

Figure 1. (A) Cross-talks between upregulated microRNAs (miRNAs) and downregulated mRNAs; and (B) downregulated miRNAs and upregulated mRNAs. 
Table V. Downregulated mRNAs in signaling pathways.

\begin{tabular}{|c|c|c|}
\hline Pathway & Enrichment score & Genes \\
\hline Transcriptional misregulation in cancer & 2.1658 & GOLPH3L//H3F3A//H3F3B//JMJD1C//MEF2C//RUNX1T1 \\
\hline Dopaminergic synapse & 2.065915 & GNAS//GNB1//KCNJ6//PPP2R1B//PPP2R2A \\
\hline Insulin secretion & 2.029172 & ATP1A3//CCKAR//GNAS//KCNMA1 \\
\hline Morphine addiction & 1.945851 & ADRBK1//GNAS//GNB1//KCNJ6 \\
\hline Alcoholism & 1.939052 & GNAS//GNB1//H3F3A//H3F3B//NTRK2//SOS1 \\
\hline Endometrial cancer & 1.880095 & $I L K / / S O S 1 / / T C F 7 L 2$ \\
\hline Hippo signaling pathway & 1.809569 & $L A T S 1 / / P P P 2 R 1 B / / P P P 2 R 2 A / / T C F 7 L 2 / / Y W H A B$ \\
\hline Acute myeloid leukemia & 1.772872 & RUNX1T1//SOS1//TCF7L2 \\
\hline Pancreatic secretion & 1.752665 & ATP1A3//CCKAR//GNAS//KCNMA1 \\
\hline Dorso-ventral axis formation & 1.736718 & SOS1//SPIRE2 \\
\hline mTOR signaling pathway & 1.694671 & RRAGD//STK11//STRADA \\
\hline Viral carcinogenesis & 1.66536 & ACTN1//DDX3X//H2-Q6//IRF9//SCIN//YWHAB \\
\hline Salivary secretion & 1.433264 & ATP1A3//GNAS//KCNMA1 \\
\hline FoxO signaling pathway & 1.402263 & GABARAPL1//GADD45A//SOS1//STK11 \\
\hline Hepatitis C & 1.392344 & $I R F 9 / / P P P 2 R 1 B / / P P P 2 R 2 A / / S O S 1$ \\
\hline PI3K-Akt signaling pathway & 1.314372 & GNB1//PPP2R1B//PPP2R2A//SOS1//SPP1//STK11//YWHAB \\
\hline Gap junction & 1.30117 & GNAS//MAP2K5//SOS1 \\
\hline
\end{tabular}

mTOR, mammalian target of rapamycin; FoxO, forkhead box O; PI3K-Akt, phosphatidylinositol 3-kinase-protein kinase B.

Table VI. Upregulated mRNAs in signaling pathways.

\begin{tabular}{lll}
\hline Pathway & Enrichment score & \multicolumn{1}{c}{ Genes } \\
\hline Axon guidance & 1.821244 & GNAI2//LIMK1//ROBOI//ROBO2 \\
Rap1 signaling pathway & 1.668166 & GNAI2//PLCB2//RALA//SIPA1L3//TIAM1 \\
Pentose and glucuronate interconversions & 1.583815 & KL//UGDH \\
Estrogen signaling pathway & 1.445183 & $C R E B 5 / / G N A I 2 / / P L C B 2$ \\
Melanogenesis & 1.423059 & GNAI2//PLCB2//WNT7B \\
Retrograde endocannabinoid signaling & 1.390859 & GNAI2//NAPEPLD//PLCB2 \\
Nucleotide excision repair & 1.359557 & ERCC4//POLE2 \\
Huntington's disease & 1.341732 & ATP5F1//CREB5//PLCB2//PPARGC1A \\
Amino sugar and nucleotide sugar metabolism & 1.308973 & $P M M 1 / / U G D H$ \\
\hline
\end{tabular}

Rap1, Ras-proximate-1.

miR-26a-5p, miR-26b-5p, miR-27a-3p and miR-148b-3p compared to the normal controls. These results suggested that a decreased miRNA profile may indicate the decreased islet $\beta$-cell number in diabetic mice. $m i R-143-3 p$ was suggested as an miRNA with high abundance in islet cells, but outside the islet $\beta$-cell (10). In the present study, miR-143-3p showed a significant decrease in pancreatic tissues of diabetic mice, which indicated that besides islet $\beta$-cells, STZ-induced diabetic mice showed a reduction in other islet cells. $m i R-184$ and $m i R-182-5 p$, considered as human islet-specific miRNA (10), were also reduced in the pancreatic tissues of STZ-induced diabetic mice compared to the normal controls, which suggested that these miRNAs may be able to serve as markers indicating islet cell number. Besides this, the roles these changed miRNAs play remains unclear.
miRNAs can target mRNAs and inhibit the gene transcriptions and protein translations. In the present study, the target mRNAs of the miRNAs were predicted, and numerous fluctuating expressions between miRNAs and targeted mRNAs were identified. These changed miRNAs may contribute to these changed target miRNAs. For insulin or pancreatic secretion: Downregulation of ATP1A3, CCKAR, GNAS and KCNMA1 were observed in pancreatic tissues in diabetic mice. These factors may be associated with impaired extracellular calcium uptake and impaired intracellular calcium release from ER (http://www.kegg.jp/kegg-bin/highlight_pathway?scale $=1.0 \&$ map=map04911\&keyword=insulin secretion). Impaired extracellular calcium uptake and impaired intracellular calcium release from the ER may contribute to impaired insulin and pancreatic secretion $(12,13) . P L C$ and $C R E B$ were associated 
Table VII. Cross-talks of miRNA-mRNA in the insulin or pancreatic secretion pathway.

\begin{tabular}{lcccc}
\hline miRNAs & Change (fold) & Gene & Change (fold) & Functions \\
\hline mmu-miR-705 & 2.5403969 & ATP1A3 & -2.183364 & Extracelluar calcium intake $\downarrow$ \\
$m$ mu-miR-450a-2-3p & 2.0858644 & $C C K A R$ & -2.5761988 & Intracelluar calcium release $\downarrow$ \\
$m m u-m i R-30 b-3 p$ & 2.1181663 & GNAS & -4.540604 & Intracelluar calcium release $\downarrow$ \\
$m$ mu-miR-139-5p & 3.1423193 & KCNMA1 & -2.322966 & Extracelluar calcium intake $\downarrow$ \\
$m$ mu-miR-669m-3p & 3.5794668 & & &
\end{tabular}

miRNA and miR, microRNA; $\downarrow$, downregulation.

Table VIII. Cross-talks of miRNA-mRNA in the FoxO signaling pathway.

\begin{tabular}{|c|c|c|c|c|}
\hline miRNAs & Change (fold) & Gene & Change (fold) & Functions \\
\hline$m m u-m i R-323-3 p$ & 3.7514458 & SOS1 & -2.0607998 & (-) FoxO by MAPK signaling pathway $\downarrow$ \\
\hline$m m u-m i R-369-3 p$ & 2.0913764 & & & \\
\hline mmu-miR-302a-3p & 2.0039108 & STK11 & -2.365607 & $(+)$ FoxO by AMPK $\downarrow$ \\
\hline$m m u-m i R-302 b-5 p$ & 2.1082705 & $G A D D 45 A$ & -2.2060218 & Repair of DNA damage $\downarrow$ \\
\hline$m m u-m i R-491-5 p$ & 3.3445722 & & & \\
\hline mmu-miR-133a-3p & 2.0759213 & GABARAPL1 & -2.1953428 & Autophagy $\downarrow$ \\
\hline$m m u-m i R-133 b-3 p$ & 2.0765676 & & & \\
\hline mmu-miR-1956 & 2.1485944 & & & \\
\hline
\end{tabular}

miRNA and miR, microRNA; FoxO, forkhead box O; MAPK, mitogen-activated protein kinase; AMPK, adenosine monophoshate-activated protein kinase; +, activation; -, inhibition; $\downarrow$, downregulation.

Table IX. Cross-talks of miRNA-mRNA in the PI3K-Akt signaling pathway.

\begin{tabular}{|c|c|c|c|c|}
\hline miRNAs & Change (fold) & Gene & Change (fold) & Functions \\
\hline$m m u-m i R-139-5 p$ & 3.1423193 & GNB1 & -2.3987343 & (+) PI3K class $1 \mathrm{~b} \downarrow$ \\
\hline$m m u-m i R-881-3 p$ & 5.5111446 & $S P P 1$ & -2.0848067 & (+) PI3K class $1 \mathrm{a} \downarrow$ \\
\hline$m m u-m i R-337-3 p$ & 2.6351878 & $Y W H A B$ & -2.2295 & $(-) \mathrm{FoxO} \downarrow$ \\
\hline$m m u-m i R-323-3 p$ & 3.7514458 & SOS1 & -2.0607998 & (+) PI3K Class 1a by Ras $\downarrow$ \\
\hline$m m u-m i R-369-3 p$ & 2.0913764 & & & \\
\hline mmu-miR-1956 & 2.1485944 & $P P P 2 R 1 B$ & -2.0079954 & $(-) \mathrm{Akt}$ \\
\hline$m m u-m i R-1956$ & 2.1485944 & $P P P 2 R 2 A$ & -2.852652 & $(-) \mathrm{Akt}$ \\
\hline$m m u-m i R-668-3 p$ & 3.0493513 & & & \\
\hline$m m u-m i R-881-3 p$ & 5.5111446 & & & \\
\hline
\end{tabular}

miRNA and miR, microRNA; PI3K-Akt, phosphatidylinositol 3-kinase-protein kinase B; FoxO, forkhead box O; +, activation; -, inhibition; $\downarrow$, downregulation.

with the promotion of insulin secretion and synthesis $(14,15)$. In the present study, $P L C$ and $C R E B$ were upregulated in the pancreatic tissues of diabetic mice. Therefore, for insulin secretion, although it is impaired, it still has compensatory responses to this impairment. Upregulation of $m m u-m i R-705$, mmu-miR-450a-2-3p, mmu-miR-30b-3p, mmu-miR-139-5p and $m m u-m i R-669 m-3 p$ may contribute to the downregulation of those factors, respectively.

For the FoxO signaling pathway, STK11 activates 5 ' adenosine monophosphate-activated protein kinase (AMPK) and subsequently activates FoxO, and may inhibit cell apoptosis (http://www.kegg.jp/kegg-bin/highlight_pathway?scale= $1.0 \&$ map $=$ map04068\&keyword=Foxo1). However, SOS1 activates mitogen-activated protein kinases signaling pathway and subsequently inhibits FoxO, and may promote cell apoptosis. In the present study, the effect of STK11 downregulation may exceed the effect of SOS1 downregulation, showed reduced FoxO and subsequently promoted islet $\beta$-cell apoptosis in diabetic mice. GADD45A mediated the repair of DNA damage in islet cells (16). Decreased expression of GADD45A indicated 
Table X. Cross-talks of miRNA-mRNA in the mTOR signaling pathway.

\begin{tabular}{lcccc}
\hline miRNAs & Change (fold) & Gene & Change (fold) & Functions \\
\hline mmu-miR-302a-3p & 2.0039108 & STK11 & -2.365607 & $(+)$ Autophagy $\downarrow$ \\
mmu-miR-1953 & 2.2295675 & STRADA & -2.7249522 & $(+)$ Autophagy $\downarrow$ \\
$m$ mu-miR-302a-3p & 2.0039108 & RRAGD & -2.0429912 & $(-)$ Autophagy
\end{tabular}

miRNA and miR, microRNA; mTOR, mammalian target of rapamycin; +, activation; -, inhibition; $\downarrow$, downregulation.

Table XI. miRNA-mRNA cross talks in the pancreatic islets of streptozotocin-induced diabetic mice.

\begin{tabular}{|c|c|c|c|}
\hline miRNAs & mRNA & Signaling pathways & Biological functions \\
\hline$m i R-705 \uparrow$ & $A T P 1 A 3 \downarrow$ & Insulin secretion $\downarrow$ & Insulin secretion $\downarrow$ \\
\hline$m i R-450 a-2-3 p \uparrow$ & $C C K A R \downarrow$ & Insulin secretion $\downarrow$ & Insulin secretion $\downarrow$ \\
\hline$m i R-30 b-3 p \uparrow$ & $G N A S \downarrow$ & Insulin secretion $\downarrow$ & Insulin secretion $\downarrow$ \\
\hline$m i R-139-5 p \uparrow$ & KCNMAl & Insulin secretion $\downarrow$ & Insulin secretion $\downarrow$ \\
\hline \multicolumn{4}{|l|}{$m i R-669 m-3 p \uparrow$} \\
\hline$m i R-302 a-3 p \uparrow$ & $S T K 11 \downarrow$ & PI3K-Akt $\downarrow$ & Apoptosis $\uparrow$ \\
\hline$m i R-302 b-5 p \uparrow$ & $G A D D 45 A \downarrow$ & PI3K-Akt $\downarrow$ & Repair of DNA damage $\downarrow$ \\
\hline \multicolumn{4}{|l|}{$m i R-491-5 p \uparrow$} \\
\hline$m i R-133 a-3 p \uparrow$ & $G A B A R A P L 1 \downarrow$ & PI3K-Akt $\downarrow$ & Autophagy $\downarrow$ \\
\hline \multicolumn{4}{|l|}{$m i R-133 b-3 p \uparrow$} \\
\hline \multicolumn{4}{|l|}{$\operatorname{miR}-1956 \uparrow$} \\
\hline $\operatorname{miR}-1956 \uparrow$ & $P P P 2 R 1 B \downarrow$ & $\mathrm{FoxO} \downarrow$ & Apoptosis $\uparrow$ \\
\hline $\operatorname{miR}-1956 \uparrow$ & $P P P 2 R 2 A \downarrow$ & FoxO $\downarrow$ & Apoptosis $\uparrow$ \\
\hline \multicolumn{4}{|l|}{$m i R-668-3 p \uparrow$} \\
\hline \multicolumn{4}{|l|}{$m i R-881-3 p \uparrow$} \\
\hline$m i R-302 a-3 p \uparrow$ & STK11 11 & mTOR $\downarrow$ & Autophagy $\downarrow$ \\
\hline $\operatorname{miR}-1953 \uparrow$ & $S T R A D A \downarrow$ & mTOR $\downarrow$ & Autophagy $\downarrow$ \\
\hline
\end{tabular}

miRNA and miR, microRNA; $\uparrow$, upregulation; $\downarrow$, downregulation; PI3K-Akt, phosphatidylinositol 3-kinase-protein kinase B; FoxO, forkhead box $\mathrm{O}$; mTOR, mammalian target of rapamycin.

that diabetic mice showed enhanced DNA damage in pancreatic islet cells. Diabetic mice show an impaired autophagy in islet cells (17). GABARAPL1 indicated the formation of autophagy. Decreased GABARAPL1 suggested that diabetic mice showed a decreased autophagy in pancreatic islet cells. mmu-miR-302a-3p, mmu-miR-302b-3p/mmu-miR-491-5p and mmu-miR-133a-3p/mmu-miR-133b-3p/mmu-miR-1956 may contribute to the downregulation of STK11, GADD45A and GABARAPL1, respectively.

For the PI3K-Akt signaling pathway, GNB1, SPP1 and SOS1 can activate PI3K and subsequently activate Akt (http://www. kegg.jp/kegg-bin/highlight_pathway?scale=1.0\&map=map041 51 \&keyword=PI3k-akt). Akt can inhibit FoxO (18). In addition, YWHAB directly inhibits FoxO. FoxO1 regulates $\beta$-cell proliferation and protects against $\beta$-cell failure induced by oxidative stress through NeuroD and MafA induction. Subsequently, the inhibition of FoxO can cause cell failure (19). In the present study, the decrease in GNB1, SPP1, SOS1 and $Y W H A B$ may upregulate FoxO and subsequently inhibit apoptosis in pancreatic $\beta$-cells of diabetic mice. Despite this, downregulation of $P P P 2 R 1 B$ or $P P P 2 R 2 A$ may attenuate the inhibition effect on $A K T$ and subsequently inhibit the expression of FoxO. The function of FoxO was decreased in pancreatic islet cells in diabetic mice (20). Therefore, the mixed effect of $P P P 2 R 1 B$ and PPP2R2A downregulation may be more than that of GNB1, SPP1, SOS1 and YWHAB. mmu-miR-1956 and mmu-miR-1956/mmu-miR-668-3p/mmu-miR-668-3p may contribute to the downregulation of $P P P 2 R 1 B$ and $P P P 2 R 2 A$, respectively.

For the mTOR signaling pathway, STK11 and STRADA activate AMPK and subsequently inhibit the mTOR signaling pathway and promote autophagy (http://www.kegg.jp/keggbin/highlight_pathway?scale=1.0\&map=map04150\&keyword= mtor). RRAGD directly activates the mTOR signaling pathway and subsequently inhibits the autophagy. In the present study, the effect of downregulation of the mixed effect of STK11 and STRADA downregulation may be stronger than that of RRAGD and may show an impaired autophagy in pancreatic islet cells of STZ-induced diabetic mice. Downregulation of RRAGD may just be a homeostasis response to this downregulation of 
autophagy in the islet cells of diabetic mice. mmu-miR-302a-3p and $m m u-m i R-1953$ may contribute to the downregulation of STK11 and STRADA, respectively.

However, for downregulated miRNAs and upregulated mRNAs, a specific significant pathway that was directly involved in the pancreatic function and development of diabetes was not identified. Those changed pathways require further validation in future studies. Regardless, numerous upregulated miRNA were identified to have certain potential cross-talks over targeted mRNAs as described above (Table XI). Among these pathways, notably, certain mRNAs showed reverse functions and indicated that these pathways can be self-regulated to maintain cell or tissue homeostasis. This homeostasis response may be useful to cause self-restoration of pancreatic islet cells in STZ-induced diabetic mice.

In conclusion, the present study firstly showed that miRNAs and matched mRNAs may have a wide cross-talk in pancreatic islet cells in diabetic mice. Insulin and pancreatic secretion, and FoxO, PI3K-Akt and the mTOR signaling pathways may be regulated by corresponding miRNAs and contribute to the development of diabetes. miRNA may cause dysfunctions of these pathways, which were associated with impaired calcium release and insulin secretion, increased DNA damage and apoptosis, and decreased autophagy in pancreatic islet cells of diabetic mice. However, further validation is required in future studies.

\section{Acknowledgements}

The present study was supported by the National Natural Science Foundation of China (grant nos. 81373460 and 81072680), the Guangdong Natural Science Foundation (grant no. 1414050003849) and the Shenzhen Science and Technology R\&D Foundation (grant nos. SGLH20121008144756945 and ZYC201105170341A).

\section{References}

1. Kameswaran V and Kaestner KH: The Missing lnc(RNA) between the pancreatic $\beta$-cell and diabetes. Front Genet 5: 200, 2014.

2. Wajchenberg BL: beta-cell failure in diabetes and preservation by clinical treatment. Endocr Rev 28: 187-218, 2007.

3 . Hur KY, Jung HS and Lee MS: Role of autophagy in $\beta$-cell function and mass. Diabetes Obes Metab 12 (Suppl 2): 20-26, 2010.

4. Roep BO and Tree TI: Immune modulation in humans: Implications for type 1 diabetes mellitus. Nat Rev Endocrinol 10: $229-242,2014$
5. Eliasson L and Esguerra JL: Role of non-coding RNAs in pancreatic beta-cell development and physiology. Acta Physiol (Oxf) 211: 273-284, 2014

6. Bartel DP: MicroRNAs: Genomics, biogenesis, mechanism and function. Cell 116: 281-297, 2004.

7. Zeng LQ, Wei SB, Sun YM, Qin WY, Cheng J, Mitchelson K and Xie L: Systematic profiling of mRNA and miRNA expression in the pancreatic islets of spontaneously diabetic Goto-Kakizaki rats. Mol Med Rep 11: 67-74, 2015.

8. Liao X, Xue H, Wang YC, Nazor KL, Guo S, Trivedi N, Peterson SE, Liu Y, Loring JF and Laurent LC: Matched miRNA and mRNA signatures from an hESC-based in vitro model of pancreatic differentiation reveal novel regulatory interactions. J Cell Sci 126: 3848-3861, 2013.

9. Naderi E, Mostafaei M, Pourshams A and Mohamadkhani A: Network of microRNAs-mRNAs interactions in pancreatic cancer. Biomed Res Int 2014: 534821, 2014.

10. van de Bunt M, Gaulton KJ, Parts L, Moran I, Johnson PR, Lindgren CM, Ferrer J, Gloyn AL and McCarthy MI: The miRNA profile of human pancreatic islets and beta-cells and relationship to type 2 diabetes pathogenesis. PLoS ONE 8: e55272, 2013.

11. Xie W, Zhao Y, Gu D, Du L, Cai G and Zhang Y: Scorpion in combination with gypsum: Novel antidiabetic activities in Streptozotocin-Induced diabetic mice by Up-Regulating pancreatic PPAR $\gamma$ and PDX-1 expressions. Evid Based Complement Alternat Med 2011: 683561, 2011.

12. Gilon P, Chae HY, Rutter GA and Ravier MA: Calcium signaling in pancreatic $\beta$-cells in health and in Type 2 diabetes. Cell Calcium 56: 340-361, 2014

13. Félix-Martínez GJ and Godínez-Fernández JR: Mathematical models of electrical activity of the pancreatic $\beta$-cell: A physiological review. Islets 6: e949195, 2014.

14. Kim HS, Hwang YC, Koo SH, Park KS, Lee MS, Kim KW and Lee MK: PPAR- $\gamma$ activation increases insulin secretion through the up-regulation of the free fatty acid receptor GPR40 in pancreatic $\beta$-cells. PLoS ONE 8: e50128, 2013.

15. Barker CJ, Li L, Kohler M and Berggren PO: $\beta$-Cell $\mathrm{Ca}\left(2^{+}\right)$ dynamics and function are compromised in aging. Adv Biol Regul 57: 112-119, 2015.

16. Hughes KJ, Meares GP, Chambers KT and Corbett JA: Repair of nitric oxide-damaged DNA in beta-cells requires JNK-dependent GADD45alpha expression. J Biol Chem 284: 27402-27408, 2009.

17. Lee MS: Role of islet $\beta$ cell autophagy in the pathogenesis of diabetes. Trends Endocrinol Metab 25: 620-627, 2014.

18. Tsai WC, Bhattacharyya N, Han LY, Hanover JA and Rechler MM: Insulin inhibition of transcription stimulated by the forkhead protein Foxol is not solely due to nuclear exclusion. Endocrinology 144: 5615-5622, 2003.

19. Buteau J and Accili D: Regulation of pancreatic beta-cell function by the forkhead protein FoxO1. Diabetes Obes Metab 9 (Suppl 2): 140-146, 2007.

20. Kim-Muller JY, Zhao S, Srivastava S, et al: Metabolic inflexibility impairs insulin secretion and results in MODY-like diabetes in triple FoxO-deficient mice. Cell Metab 20: 593-602, 2014. 